\title{
Optimal trajectory generation with direct acceleration reshaping for autonomous vehicles
}

\author{
Isao OKAWA*, Yoshihide MIZUSHIMA** and Kenichiro NONAKA*** \\ * Advanced Mobility Systems R\&D Division, DENSO CORPORATION \\ W Building 17F, 1-8-15 Konan, Minato, Tokyo 108-0075, Japan \\ E-mail: isao.okawa.j3g@jp.denso.com \\ ** Mechanics, Graduate School of Integrative Science and Engineering, Tokyo City University \\ 1-28-1 Tamazutsumi, Setagaya, Tokyo 158-8557, Japan \\ *** Department of Mechanical Systems Engineering, Tokyo City University \\ 1-28-1 Tamazutsumi, Setagaya, Tokyo 158-8557, Japan
}

Received: 9 December 2019; Revised: 30 April 2020; Accepted: 11 June 2020

\begin{abstract}
This paper focuses on acceleration trajectory shaping using model predictive control for autonomous vehicles. The proposed method employs two types of constraints for the shaping: hard constraints, which must be satisfied and soft constraints, which can be relaxed if required. The soft constraints require that the acceleration trajectory be shaped into the desired piece-wise linear function of time, while collision avoidance is guaranteed by utilizing hard constraints. Since we can specify the desired level of acceleration and jerk directly, it becomes straightforward to design and adjust the shape of the trajectory. Further, fast and stable solvers are available, since the optimization problem is formulated in convex quadratic programming. We employ a desired trajectory with constant acceleration (deceleration) as a typical target, and validate the reshaping performance and verify the feasibility of the method through experiments with real vehicles. Two experimental scenarios are considered to ensure the compatibility of trajectory shaping and collision avoidance: sudden braking of a preceding vehicle and cutting-in by a slow-moving vehicle. The experimental results show that the proposed method successfully shaped the trajectory satisfying collision avoidance, while soft constraints for shaping were appropriately relaxed as demanded, which supports the effectiveness of the proposed method.
\end{abstract}

Keywords : Motion control, Optimal control, Optimization problem techniques, Automobiles and industrial vehicles, Intelligence and autonomy technology

\section{Introduction}

Autonomous driving technologies have been actively studied and developed for application on urban roads following successful studies on highways (Aeberhard et al., 2015) (Ziegler et al., 2014). Whereas highway operation basically consists of constant speed cruising and following the preceding vehicle by mild acceleration / deceleration, for operation on urban roads it is additionally necessary to cope with traffic lights, sudden braking by a preceding vehicle, cuttingin by other vehicles, and other issues such as the movements of pedestrians. Thus, frequent and non-mild acceleration / deceleration is imperative and collision avoidance in such environments becomes a necessary requirement for applications on urban roads. However, collision avoidance may frequently cause unsuitable braking operations for passengers vis-à-vis their comfort and thus complicates popularization of the technology. Therefore, generating a suitable target trajectory for passengers is a significant task pursuant of the successful application of autonomous driving on urban roads (Wang et al., 2000).

Adaptive cruise control (ACC) is a common function for autonomous speed control (Persson et al., 1999) (Vahidi and Eskandarian, 2003) and has been studied with respect to suppressed acceleration or jerk considering suitability for passengers (Ioannou and Xu, 1994) (Martinez and Wit, 2007) (Yamamura et al., 2008). To realize collision avoidance 
and ensure dynamic performance, applications of model predictive control (MPC), have been studied (Bageshwar et al., 2004) (Naus et al., 2008) because this allows explicit consideration for constraints. These applications employ constraints for relative distance between the preceding vehicle and the ego vehicle to prevent collision, while upper and lower bound constraints for acceleration and jerk are invoked to suppress their magnitude. Moreover, studies that explicitly consider cutting-in by another vehicle in the context of smooth acceleration or deceleration (Liu et al., 2017) and appropriate parameter settings on the Criterion function (Zhao et al., 2017) have also been reported.

However, since constraints on relative distance, acceleration and jerk sometimes contradict each other, it is difficult to guarantee feasibility for situations in which heavy braking is required to avoid a collision. To overcome these difficulties, studies have explored relaxing the constraints for relative distance (Kianfar et al., 2018) (Stanger and Del Re, 2013) and acceleration ( $\mathrm{Li}$ et al., 2009) (Junhui et al., 2018). These methods realize collision avoidance as well as suppression of acceleration, by relaxing the constraints when necessary to guarantee feasibility. However, since the shape of the generated trajectory is not directly designed, there remain possibilities for causing vehicle behavior, which passengers would deem undesirable.

Based on our previous work (Mizushima et al., 2019), in this paper we propose a trajectory shaping method which directly designs the shape of the trajectory into a desired piece-wise linear function of time. The proposed method enables the trajectory to be adjustable in compliance with each user's desired specification. We assume that the velocity reference is generated by the integration of the following two commonly used control functions, cruise control (CC) and adaptive cruise control (ACC). Consideration of (quasi-) static environment parameters such as the legal speed limit and traffic signals is covered by CC whilst dynamic phenomena such as other vehicles are addressed by ACC. Then, we apply constrained MPC for the reference trajectory to reshape it so that the acceleration trajectory is designed in accordance with satisfying the constraints. In the proposed method, requirements vis-à-vis safety and legality are adopted as hard constraints which must be satisfied, while requirements concerning suitability for passengers like suppressed acceleration and jerk are adopted as soft constraints which are relaxed if necessary. Applying the MPC, sufficiency for the critical requirements are guaranteed, and feasibility can be determined. Thus, the proposed method can ensure the driving safety and suitable response on urban roads, where numerous situations are expected. To configure the soft constraints to be compliant with the desired level of acceleration and jerk, the acceleration trajectory is shaped into a corresponding piecewise linear function of time. Thus, collision avoidance and direct trajectory shaping can be achieved.

For real world applications, it is necessary to validate real-time computation performance to solve constrained MPC with iterations, and robustness for the adverse road condition like, for example, slippery surface. In our preceding study (Mizushima et al., 2019), the effectiveness of the proposed method was validated through simulations, however, the computation performance and the robustness for the road condition were not accommodated. Thus, herein, we validate the effectiveness of the method through experiments with real vehicles. Two experimental scenarios are adopted as examples to explore collision avoidance and trajectory shaping: sudden braking by the preceding vehicle and cutting-in by a slow-moving vehicle. Through these results we demonstrate the effectiveness of the method.

\section{Autonomous driving system}

\subsection{System overview}

Figure 1 depicts the autonomous driving system for distance, speed and acceleration control considered in this study. The system generally consists of the trajectory generator and the tracking controller. The trajectory generator computes optimal state trajectories corresponding to the driving environment, then the tracking controller ensures that the actual vehicle follows the generated trajectories. In Fig. 1, the trajectory generator consists of CC, ACC, integration function, MPC and motion model of the ego vehicle. CC and ACC generate reference trajectories and constraints for MPC. CC concerns tasks for solo driving such as constant speed cruising and reaction to traffic signals. ACC focuses on tasks for accommodative driving such as deceleration with respect to preceding or cutting-in vehicles. Based on the reference trajectories and constraints from CC and ACC, the integration function generates the integrated reference trajectories and constraints for MPC. Then, applying the optimal input trajectory computed by MPC to the motion model, the optimal state trajectories are obtained. The tracking controller computes the control input to track the optimal state trajectories and drives the vehicle. Repeating the above sequence within a given control interval, the optimal vehicle behavior corresponding to the driving environment is realized.

\subsection{Vehicle motion model}

Since the target of this study is to reshape the acceleration trajectory, we derive the vehicle motion model along the 


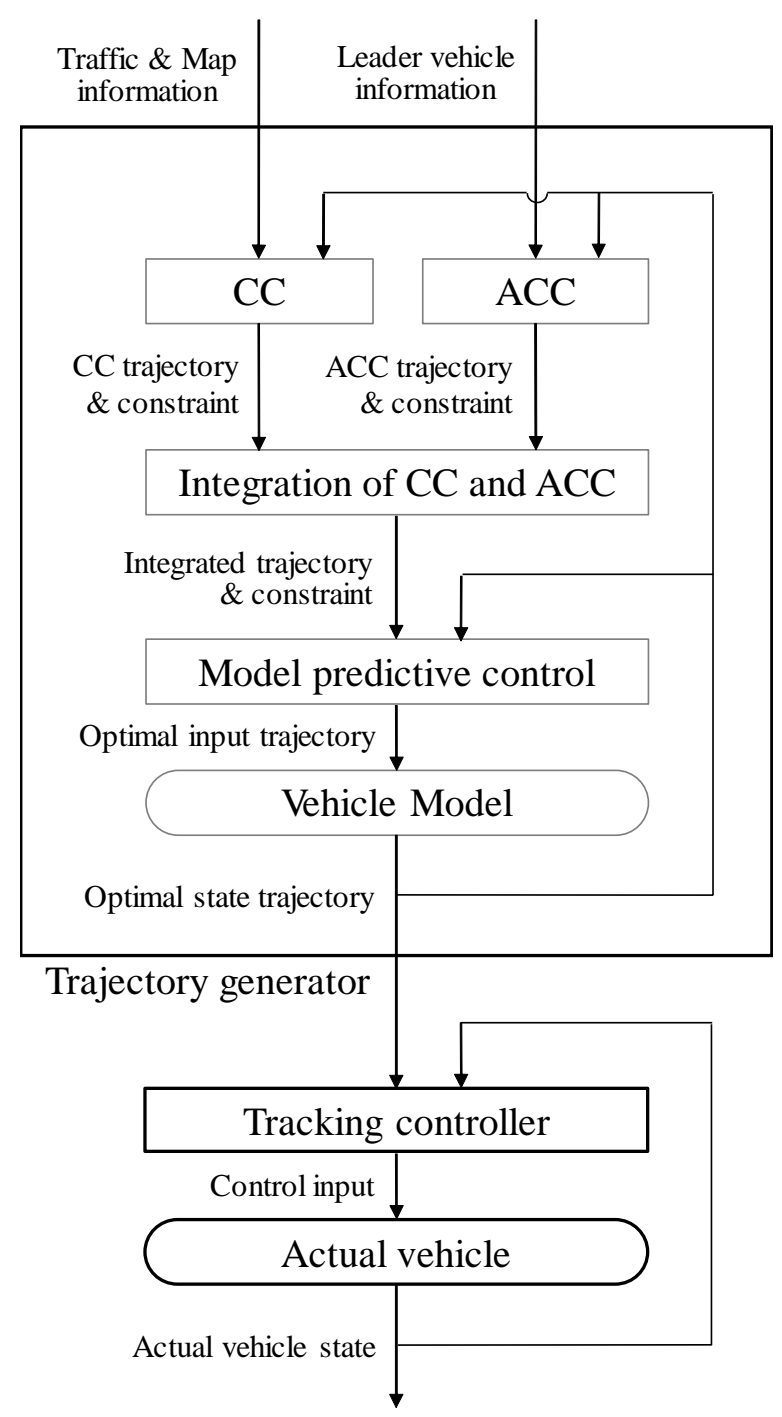

Fig. 1 The autonomous driving system for distance, speed and acceleration control. The system consists of two major functions: trajectory generator and tracking controller. The trajectory generator computes the optimal state trajectory as a favorable vehicle behavior, while the tracking controller realizes the generated optimal state.

direction of travel. We define the state vector $\boldsymbol{x}:=[d, v, a]^{T}$ and the input $u:=j$, where $d, v, a$ and $j$ represent distance $[\mathrm{m}]$, speed $[\mathrm{m} / \mathrm{s}]$, acceleration $\left[\mathrm{m} / \mathrm{s}^{2}\right]$ and jerk $\left[\mathrm{m} / \mathrm{s}^{3}\right]$, respectively. Thus, the control object is a continuous-time triple integrator: $\dot{d}=v, \dot{v}=a$ and $\dot{a}=j$. Applying the zero-order hold with time interval $\Delta$ [s], the state equation, which is employed as the motion model of the ego vehicle, is obtained as follows:

$$
\boldsymbol{x}[k+1]=\boldsymbol{A} \boldsymbol{x}[k]+\boldsymbol{b} u[k], \quad \boldsymbol{A}:=\left[\begin{array}{ccc}
1 & \Delta & \Delta^{2} / 2 \\
0 & 1 & \Delta \\
0 & 0 & 1
\end{array}\right], \quad \boldsymbol{b}:=\left[\begin{array}{c}
\Delta^{3} / 6 \\
\Delta^{2} / 2 \\
\Delta
\end{array}\right] .
$$

It is noted that, for the roads with a small curvature, Eq. (1) is still valid if the tracking error is kept sufficiently small by exploiting the time-state control form (Okawa and Nonaka, 2018), which separates the longitudinal and lateral motion.

\subsection{Reference trajectory and constraints for MPC}

This section outlines the $\mathrm{CC}, \mathrm{ACC}$ and the integration function, which generate the reference trajectories and constraints for MPC.

$\mathrm{CC}$ generates the trajectories and constraints for solo driving, not considering preceding or cutting-in vehicles. Though the scope of conventional CC is constant speed cruising, this study includes acceleration and deceleration corresponding to changes in the legal speed limit and traffic signals. Since CC only concerns solo driving, the reference 
trajectories and constraints are designed considering suitability for passengers. The reference trajectories are designed by constant level acceleration (deceleration) without unnecessary acceleration changes, to guard against passengers' anxiety caused by unpredictable vehicle behavior. The constraints are configured by the running distance to a stop line and the legal speed limit, since they must be satisfied regardless of the existence of other vehicles. Figure 2 represents an example of reference trajectories and constraints by $\mathrm{CC}$, when the vehicle halts at a stop line which corresponds to a traffic signal.

ACC generates the trajectories and constraints for accommodative driving, considering dynamic objects like preceding or cutting-in vehicles. If there is no preceding vehicle, a virtual preceding vehicle which has sufficient speed and long relative distance is set. The reference trajectories are designed to track the preceding vehicle with appropriate relative distance corresponding to driving speed. Further, they are computed by a feedback control for a relative distance since precise prediction of the preceding vehicle motion is difficult. The constraints are configured by the travel distance to the appropriate relative distance between the preceding vehicle as well as the legal speed limit. Figure 3 represents an example of reference trajectories and constraints by ACC, when the vehicle decelerates and tracks a slow-moving preceding vehicle.

The integration function generates reference trajectories and constraints for MPC using the results of both CC and ACC. Figure 4 represents an example of integration of the trajectory in Fig. 2 and 3. The integration is preliminarily carried out for the constraints and reference speed trajectory, choosing more conservative values for each prediction time. Then, the distance and acceleration trajectory are generated by integrating and differentiating the reference speed trajectory to guarantee consistency with the speed trajectory.
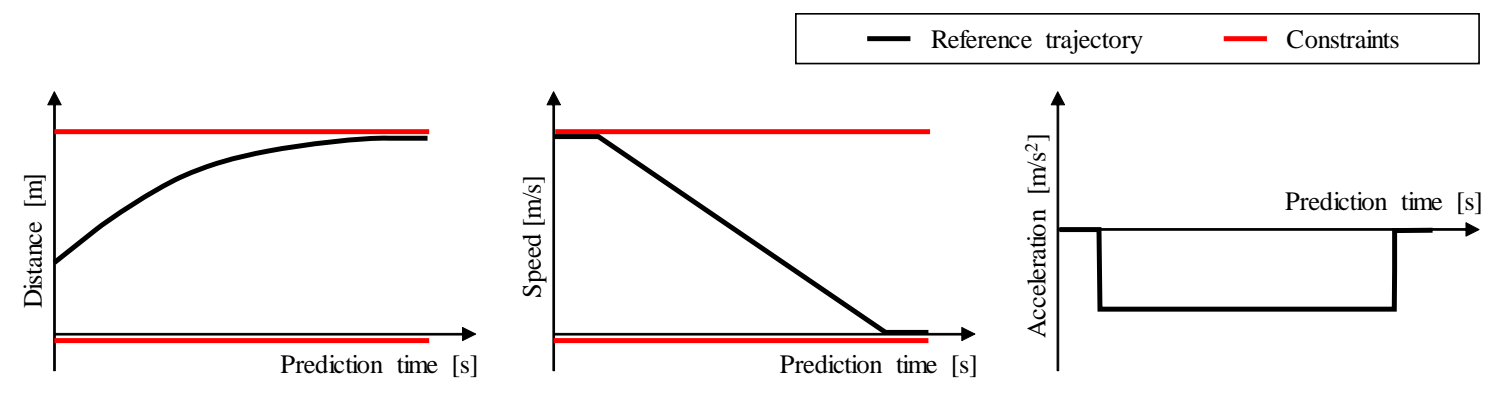

Fig. 2 Reference trajectories and constraints generated by CC to decelerate a vehicle for a stop line. The acceleration trajectory is generated as a constant-level trajectory.
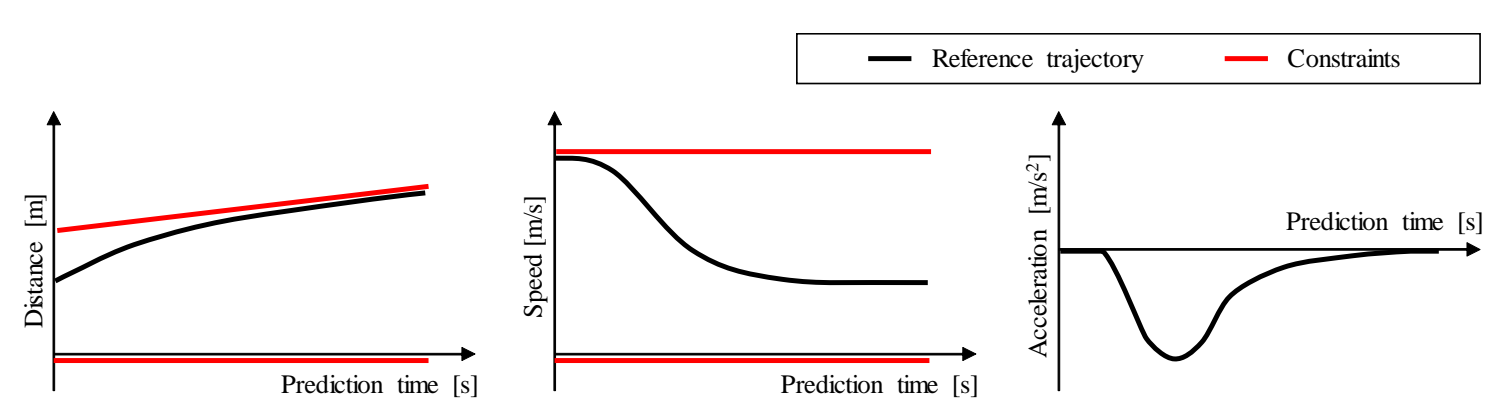

Fig. 3 Reference trajectories and constraints generated by ACC to maintain appropriate relative distance with a slow-moving preceding vehicle. The acceleration trajectory is generated as a feedback controlled trajectory.

\section{Acceleration shaping based on MPC}

\subsection{Desired trajectory (Mizushima, et al. 2019)}

In this section, the reference shape of the desired trajectory is presented. We focus on deceleration considering its importance from a safety perspective as well as highlighting the effectiveness of the proposed method. However, the method can also be applied to acceleration.

Although determining a universally preferable trajectory for passengers is difficult, since it significantly depends on personal or regional difference, it seems to be reasonable that the trajectories with unnecessary acceleration changes, excessive jerk, or languid braking should be preventable. Therefore, we consider the piecewise linear acceleration profile depicted in Fig. 5 for the desired trajectory, which comprises three sections, decrease (Range 1), hold (Range 2) and 

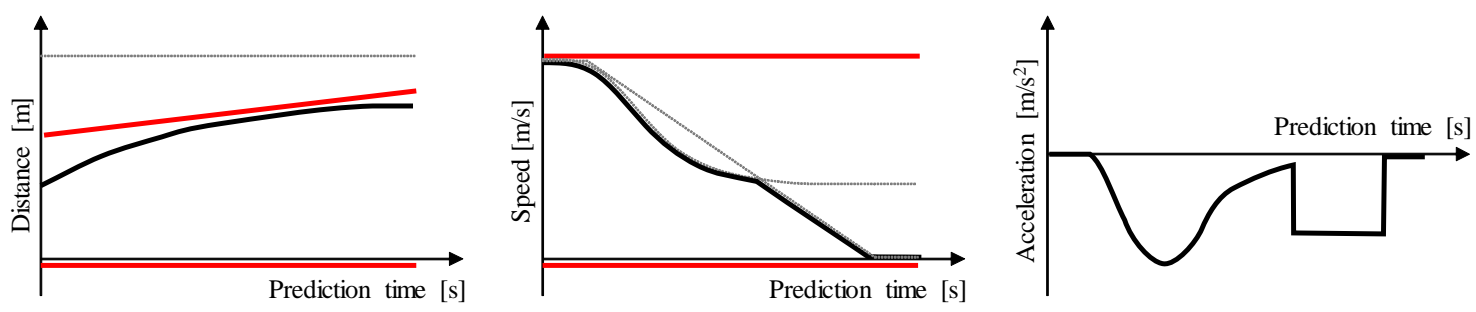

Fig. 4 Reference trajectories and constraint bounds after the integration. The reference speed trajectory and constraints are integrated choosing more conservative values at each prediction time. The distance and acceleration trajectory are generated by integrating and differentiating the reference speed trajectory.

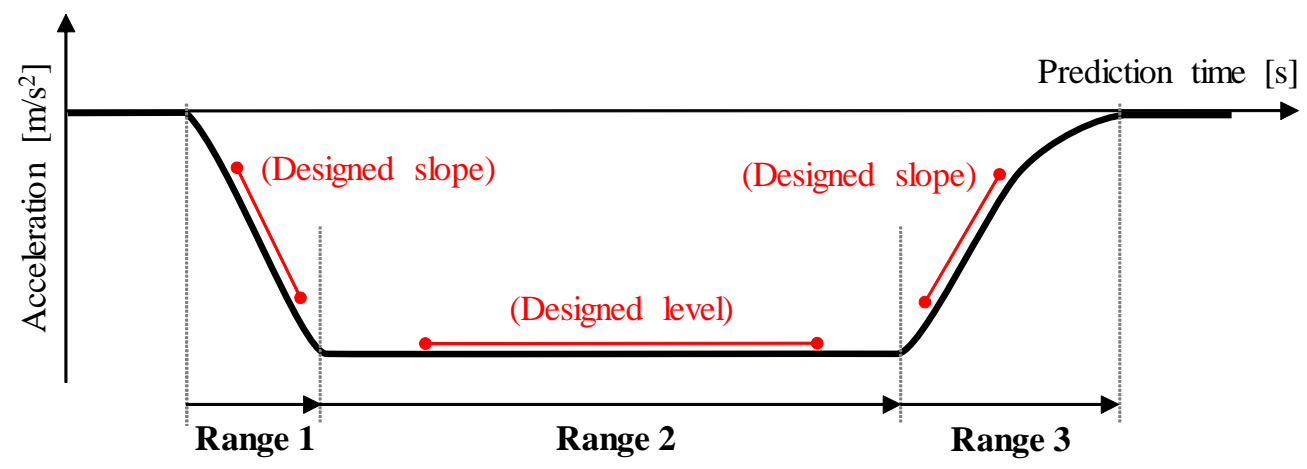

Fig. 5 The shaping target for the acceleration trajectory. The slope of Range 1 and 3, and the deceleration level of Range 2 can be designed arbitrarily.

increase (Range 3) acceleration. Range 1 and 3 can suppress excessive jerk and Range 2 can provide appropriate deceleration. The slope (jerk) of Range 1 and 3 and the constant level (acceleration) of Range 2 are assumed to be design parameters that can be calibrated by manufacturers or drivers. In the following, we present a controller that achieves this profile as long as the safety requirement is satisfied.

\subsection{Formulation of MPC (Mizushima, et al. 2019)}

This section describes the formulation of MPC, which reshapes the acceleration into the profile, as depicted in Fig. 5. We introduce two types of constraints: hard and soft. The hard constraints indicate mandatory requirements, which reflect safety for actuator limitation and collision avoidance as well as the legal requirement for adhering to the speed limit and obeying traffic signals. The soft constraint indicates the desirable requirement, which provides comfort for passengers.

\subsubsection{Constraints}

The constraints are represented as the upper and lower bound for the state $\boldsymbol{x}$ and the input $u$. To describe the hard constraints, the upper and lower bounds on travel distance $d$ are defined as $d^{u}[k]$ and $d^{l}[k]$. Both bounds on the velocity $v$ are defined as $v^{u}[k]$ and $v^{l}[k]$, and those on the acceleration $a$ are defined as $a^{u}$ and $a^{l}$ for the actuation limit to prevent excessive acceleration. Finally, those on the jerk $j$ are defined as $j^{u}$ and $j^{l}$. These hard constraints are represented as follows:

$$
\begin{array}{lll}
d^{l}[k] \leq d[k] \leq d^{u}[k] & \text { for } & k=1,2, \cdots, N, \\
v^{l}[k] \leq v[k] \leq v^{u}[k] & \text { for } & k=1,2, \cdots, N, \\
a^{l} \leq a[k] \leq a^{u} & \text { for } & k=1,2, \cdots, N, \\
j^{l} \leq j[k] \leq j^{u} & \text { for } & k=0,1, \cdots, N-1 .
\end{array}
$$

Similarly, for the soft constraints, bounds for acceleration and jerk are defined as $a_{s}^{u}$ and $a_{s}^{l}, j_{s}^{u}$ and $j_{s}^{l} . a_{s}^{u}$ and $a_{s}^{l}$ corresponds to the acceleration level of Range 2 depicted in Fig. 5, and $j_{s}^{u}$ and $j_{s}^{l}$ correspond to the slope of Range 1 and 
3, respectively. Thus, those constraints are given as follows:

$$
\begin{aligned}
& a_{s}^{l}-\sigma_{a}[k] \leq a[k] \leq a_{s}^{u}+\sigma_{a}[k] \text { for } k=1,2, \cdots, N, \\
& j_{s}^{l}-\sigma_{j}[k] \leq j[k] \leq j_{s}^{u}+\sigma_{j}[k] \quad \text { for } k=0,1, \cdots, N-1 \text {, }
\end{aligned}
$$

where $\sigma_{a}$ and $\sigma_{j}$ are slack variables for $a[k]$ and $j[k]$, which correspond to deviations from the range $\left[a_{s}^{l}, a_{s}^{u}\right]$ and $\left[j_{s}^{l}, j_{s}^{u}\right]$ for positive $\sigma_{a}$ and $\sigma_{j}$, respectively. These soft constraints accommodate the fact that $a[k]$ and $j[k]$ might stray off the ranges $\left[a_{s}^{l}, a_{s}^{u}\right]$ and $\left[j_{s}^{l}, j_{s}^{u}\right]$ for positive $\sigma_{a}$ and $\sigma_{j}$ respectively, but the deviations are minimized by evaluating them in the criterion function given in the next section.

\subsubsection{Criterion function}

The integration function depicted in Fig. 1 provides the target signals of the travel distance $d_{t}[k]$, the velocity $v_{t}[k]$ and the acceleration $a_{t}[k]$, and they configure the target state vector $\boldsymbol{x}_{t}[k]:=\left[d_{t}[k], v_{t}[k], a_{t}[k]\right]^{T}$. Then, the tracking error is given as follows:

$$
\boldsymbol{e}[k]:=\boldsymbol{x}[k]-\boldsymbol{x}_{t}[k] .
$$

Using this tracking error $\boldsymbol{e}[k]$, the Criterion function $J$ is given as follows:

$$
J:=\frac{1}{2} \boldsymbol{e}[N]^{T} \boldsymbol{Q} \boldsymbol{e}[N]+\frac{1}{2} \sum_{k=0}^{N-1}\left(\boldsymbol{e}[k]^{T} \boldsymbol{Q} \boldsymbol{e}[k]+R u[k]^{2}\right)+\frac{1}{2} Q_{a} \sum_{k=1}^{N} \sigma_{a}[k]^{2}+\frac{1}{2} Q_{j} \sum_{k=0}^{N-1} \sigma_{j}[k]^{2},
$$

where $Q, R, Q_{a}$ and $Q_{j}$ are constant matrix and scalars, and $N$ is the horizon. The first and second terms of the right-hand side of Eq. (9) denote the terminal cost and the stage cost, respectively. The third and fourth terms evaluate the magnitude of deviation concerning acceleration and jerk.

\subsection{QP formulation}

MPC is formulated as a quadratic programming $(\mathrm{QP})$ problem as follows:

$\begin{array}{llll}\text { Minimize } & J & & \\ \text { with respect to } & u[k], \sigma_{j}[k] & \text { for } & k=0,1, \cdots, N-1, \\ & \sigma_{a}[k] & \text { for } \quad k=1,2, \cdots, N\end{array}$

subject to $\quad(1),(2) \sim(7)$.

In the previous study (Mizushima et al., 2019), the above problem is directly solved using a general-purpose solver to verify the feasibility of the proposed method. In this study, the problem is transformed into another formula that is suitable for real-time computation.

The input value, state vectors, target vectors and slack variables for each prediction horizon step $k$ are aggregated into vectors as follows:

$$
\begin{aligned}
\boldsymbol{U}:=[u[0], \cdots, u[N-1]]^{T}, & \boldsymbol{X}:=\left[\boldsymbol{x}[1]^{T}, \cdots, \boldsymbol{x}[N]^{T}\right]^{T}, \quad \boldsymbol{X}_{t}:=\left[\boldsymbol{x}_{t}[1]^{T}, \cdots, \boldsymbol{x}_{t}[N]^{T}\right]^{T}, \\
\boldsymbol{S}_{a}:=\left[\sigma_{a}[1], \cdots, \sigma_{a}[N]\right]^{T}, & \boldsymbol{S}_{j}:=\left[\sigma_{j}[0], \cdots, \sigma_{j}[N-1]\right]^{T} .
\end{aligned}
$$

The weight matrix and values $Q, R, Q_{a}$ and $Q_{j}$ are also placed into the following block diagonal matrices:

$$
\begin{aligned}
& \overline{\boldsymbol{Q}}:=\operatorname{diag}(\boldsymbol{Q}, \cdots, \boldsymbol{Q}), \quad \overline{\boldsymbol{R}}:=\operatorname{diag}(R, \cdots, R), \\
& \overline{\boldsymbol{Q}}_{a}:=\operatorname{diag}\left(Q_{a}, \cdots, Q_{a}\right), \quad \overline{\boldsymbol{Q}}_{j}:=\operatorname{diag}\left(Q_{j}, \cdots, Q_{j}\right) .
\end{aligned}
$$

Then the Criterion function of Eq. (9) is represented as follows:

$$
J=\frac{1}{2}\left(\boldsymbol{X}-\boldsymbol{X}_{t}\right)^{T} \overline{\boldsymbol{Q}}\left(\boldsymbol{X}-\boldsymbol{X}_{t}\right)+\frac{1}{2}\left(\boldsymbol{x}[0]-\boldsymbol{x}_{t}[0]\right)^{T} \boldsymbol{Q}\left(\boldsymbol{x}[0]-\boldsymbol{x}_{t}[0]\right)+\frac{1}{2} \boldsymbol{U}^{T} \overline{\boldsymbol{R}} \boldsymbol{U}+\frac{1}{2} \boldsymbol{S}_{a}^{T} \overline{\boldsymbol{Q}}_{a} \boldsymbol{S}_{a}+\frac{1}{2} \boldsymbol{S}_{j}^{T} \overline{\boldsymbol{Q}}_{j} \boldsymbol{S}_{j}
$$

Using Eq. (1), $\boldsymbol{X}$ is represented by $\boldsymbol{x}[0]$ and $\boldsymbol{U}$ :

$$
\boldsymbol{X}=\bar{A} \boldsymbol{x}[0]+\overline{\boldsymbol{B}} \boldsymbol{U}, \quad \overline{\boldsymbol{A}}:=\left[\begin{array}{c}
\boldsymbol{A} \\
\vdots \\
A^{N}
\end{array}\right], \quad \overline{\boldsymbol{B}}:=\left[\begin{array}{ccc}
\boldsymbol{b} & & \boldsymbol{O} \\
\vdots & \ddots & \\
A^{N-1} b & \cdots & \boldsymbol{b}
\end{array}\right]
$$


Substituting Eq. (11) into Eq. (10) and eliminating the constant terms, another Criterion function $\bar{J}$ which has the same role as Eq. (10) is obtained.

$$
\bar{J}=\frac{1}{2} \boldsymbol{U}^{T}\left(\overline{\boldsymbol{B}}^{T} \overline{\boldsymbol{Q}} \overline{\boldsymbol{B}}+\overline{\boldsymbol{R}}\right) \boldsymbol{U}+\boldsymbol{U}^{T} \overline{\boldsymbol{B}}^{T} \overline{\boldsymbol{Q}}\left(\overline{\boldsymbol{A}} \boldsymbol{x}[0]-\boldsymbol{X}_{t}\right)+\frac{1}{2} \boldsymbol{S}_{a}^{T} \overline{\boldsymbol{Q}}_{a} \boldsymbol{S}_{a}+\frac{1}{2} \boldsymbol{S}_{j}^{T} \overline{\boldsymbol{Q}}_{j} \boldsymbol{S}_{j}
$$

In addition, $\boldsymbol{U}, \boldsymbol{S}_{a}$ and $\boldsymbol{S}_{j}$ are configured in the optimized variable $\boldsymbol{Z}$ as follows:

$$
\boldsymbol{Z}:=\left[\boldsymbol{U}^{T}, \boldsymbol{S}_{a}^{T}, \boldsymbol{S}_{j}^{T}\right]^{T}
$$

$\bar{J}$ is representable as the following form which is explicitly represented by $\mathbf{Z}$ :

$$
\begin{aligned}
& \bar{J}=\frac{1}{2} \boldsymbol{Z}^{T} \boldsymbol{H} \boldsymbol{Z}+\boldsymbol{Z}^{T} \boldsymbol{f}, \\
& \boldsymbol{H}:=\operatorname{diag}\left(\boldsymbol{H}_{o}, \overline{\boldsymbol{Q}}_{a}, \overline{\boldsymbol{Q}}_{j}\right), \quad \boldsymbol{H}_{o}:=\overline{\boldsymbol{B}}^{T} \overline{\boldsymbol{Q}} \overline{\boldsymbol{B}}+\overline{\boldsymbol{R}}, \quad \boldsymbol{f}:=\boldsymbol{F}_{o} \boldsymbol{x}[0]+\boldsymbol{F}_{t} \boldsymbol{X}_{t}, \quad \boldsymbol{F}_{o}:=\left[\begin{array}{c}
\overline{\boldsymbol{B}}^{T} \overline{\boldsymbol{Q}} \overline{\boldsymbol{A}} \\
\mathbf{0} \\
\mathbf{0}
\end{array}\right], \quad \boldsymbol{F}_{t}:=\left[\begin{array}{c}
-\overline{\boldsymbol{B}}^{T} \overline{\boldsymbol{Q}} \\
\mathbf{0} \\
\mathbf{0}
\end{array}\right]
\end{aligned}
$$

Then, the inequality constraints are also transformed into a representation which is explicit about $\boldsymbol{Z}$. First, for each prediction time of MPC, $a[k]$ are configured in the corresponding vectors as follows:

$$
\boldsymbol{X}_{3}:=[a[1], \cdots, a[N]]^{T}=\boldsymbol{E}_{3}(\overline{\boldsymbol{A}} \boldsymbol{x}[0]+\overline{\boldsymbol{B}} \boldsymbol{U}), \quad \boldsymbol{E}_{3}:=\operatorname{diag}([0,0,1], \cdots,[0,0,1]) .
$$

Next, the constraint bounds for the input and states are also configured in the corresponding vectors as follows:

$$
\begin{aligned}
& \boldsymbol{X}^{u}:=\left[\begin{array}{c}
\boldsymbol{x}^{u}[1] \\
\vdots \\
\boldsymbol{x}^{u}[N]
\end{array}\right], \quad \boldsymbol{X}^{l}:=\left[\begin{array}{c}
\boldsymbol{x}^{l}[1] \\
\vdots \\
\boldsymbol{x}^{l}[N]
\end{array}\right], \quad \boldsymbol{x}^{u}[k]:=\left[d^{u}[k], v^{u}[k], a^{u}\right]^{T}, \quad \boldsymbol{x}^{l}[k]:=\left[d^{l}[k], v^{l}[k], a^{l}\right]^{T}, \\
& \boldsymbol{U}^{u}:=j^{u} \cdot \mathbf{1}, \quad \boldsymbol{U}^{l}:=j^{l} \cdot \mathbf{1}, \quad \boldsymbol{X}_{s}^{u}:=a_{s}^{u} \cdot \mathbf{1}, \quad \boldsymbol{X}_{s}^{l}:=a_{s}^{l} \cdot \mathbf{1}, \quad \boldsymbol{U}_{s}^{u}:=j_{s}^{u} \cdot \mathbf{1}, \quad \boldsymbol{U}_{s}^{l}:=j_{s}^{l} \cdot \mathbf{1} .
\end{aligned}
$$

where $\mathbf{1} \in \mathfrak{R}^{N}$ denotes a vector in which all elements are 1 . Then, denoting the element-wise inequality by $\prec$ and $\leq$, the inequality constraints of (2) (7) are represented as follows:

$$
\begin{aligned}
& \boldsymbol{X}^{l} \leq \boldsymbol{X} \leq \boldsymbol{X}^{u}, \\
& \boldsymbol{U}^{l} \leq \boldsymbol{U} \leq \boldsymbol{U}^{u}, \\
& \boldsymbol{X}_{s}^{l}-\boldsymbol{S}_{a} \leq \boldsymbol{X}_{3} \leq \boldsymbol{X}_{s}^{u}+\boldsymbol{S}_{a}, \\
& \boldsymbol{U}_{s}^{l}-\boldsymbol{S}_{j} \leq \boldsymbol{U} \leq \boldsymbol{U}_{s}^{u}+\boldsymbol{S}_{j} .
\end{aligned}
$$

Using Eq. (11) and (14), the inequality constraints of Eq. (15) (18) are represented by the following form which is explicitly represented by $\boldsymbol{Z}$ :

$$
\tilde{A} Z \leq \tilde{b},
$$

$$
\tilde{\boldsymbol{A}}:=\left[\begin{array}{ccc}
\overline{\boldsymbol{B}} & \boldsymbol{O} & \boldsymbol{O} \\
\boldsymbol{I} & \boldsymbol{O} & \boldsymbol{O} \\
\boldsymbol{E}_{3} \overline{\boldsymbol{B}} & -\boldsymbol{I} & \boldsymbol{O} \\
\boldsymbol{I} & \boldsymbol{O} & -\boldsymbol{I} \\
-\overline{\boldsymbol{B}} & \boldsymbol{O} & \boldsymbol{O} \\
-\boldsymbol{I} & \boldsymbol{O} & \boldsymbol{O} \\
-\boldsymbol{E}_{3} \overline{\boldsymbol{B}} & -\boldsymbol{I} & \boldsymbol{O} \\
-\boldsymbol{I} & \boldsymbol{O} & -\boldsymbol{I}
\end{array}\right], \quad \tilde{\boldsymbol{b}}:=\tilde{\boldsymbol{B}}_{o} \boldsymbol{x}[0]+\boldsymbol{X}^{u l}, \quad \tilde{\boldsymbol{B}}_{o}:=\left[\begin{array}{c}
-\overline{\boldsymbol{A}} \\
\boldsymbol{O} \\
-\boldsymbol{E}_{3} \overline{\boldsymbol{A}} \\
\boldsymbol{O} \\
\bar{A} \\
\boldsymbol{O} \\
\boldsymbol{E}_{3} \overline{\boldsymbol{A}} \\
\boldsymbol{O}
\end{array}\right], \quad \boldsymbol{X}^{u l}:=\left[\begin{array}{c}
\boldsymbol{X}^{u} \\
\boldsymbol{U}^{u} \\
\boldsymbol{X}_{s}^{u} \\
\boldsymbol{U}_{s}^{u} \\
-\boldsymbol{X}^{l} \\
-\boldsymbol{U}^{l} \\
-\boldsymbol{X}_{s}^{l} \\
-\boldsymbol{U}_{s}^{l}
\end{array}\right]
$$

Therefore, MPC in the proposed method is formulated as the next QP problem.

Minimize $\quad \bar{J}=\frac{1}{2} \boldsymbol{Z}^{T} \boldsymbol{H} \boldsymbol{Z}+\boldsymbol{Z}^{T} \boldsymbol{f}$

with respect to $\quad \boldsymbol{Z}$

subject to $\quad \tilde{A} Z \leq \tilde{b}$. 
The above QP is parameterized by $\boldsymbol{H}, \boldsymbol{f}=\boldsymbol{F}_{o} \boldsymbol{x}[0]+\boldsymbol{F}_{t} \boldsymbol{X}_{t}, \tilde{\boldsymbol{A}}, \tilde{\boldsymbol{b}}=\tilde{\boldsymbol{B}}_{o} \boldsymbol{x}[0]+\boldsymbol{X}^{u l}$. Although these matrices and vectors become large as the number of prediction points $N$ increases, the matrices $\boldsymbol{H}, \boldsymbol{F}_{o}, \boldsymbol{F}_{t}, \tilde{\boldsymbol{A}}$ and $\tilde{\boldsymbol{B}}_{o}$ are time-invariant. Thus, in the online computation, the QP parameters can be easily updated by multiplying these offline-computed matrices and the other time-varying vectors. This formulation facilitates QP to be transformed into a linear complementarity problem, which is solved by finding an appropriate combination of active constraints (Okawa and Nonaka, 2018). Because the number of constraints are predetermined, certifying the maximum computational load can be determined in offline.

\subsection{Design of the constraint bounds}

The proposed method shapes the trajectory into the desired piece-wise linear function of time, by configuring the soft constraint bounds to be suitable. On the other hand, bounds for the acceleration should be determined considering the road friction to prevent a vehicle from going into a skid. However, because the road friction is perturbed depending on the insensible change in road condition, bounds concerning the road friction have to be set as sufficiently conservative. If these bounds are imposed on the hard constraints, the MPC problem may frequently become infeasible and be terminated inappropriately. To address this issue, these bounds should be represented by the soft constraints, which can be relaxed and accept perturbation of the bounds. In this section, it is shown how to design the constraint bounds, which are consistent with the requirement of road friction. The adequacy of the usage of soft constraints are experimentally validated in the next chapter.

First, a model for the acceleration bounds described by road friction coefficient is derived. Let $\mu, g, a_{n}$ be the friction coefficient $[\mathrm{ND}]$, gravity acceleration $\left[\mathrm{m} / \mathrm{s}^{2}\right]$, vehicle turning lateral acceleration $\left[\mathrm{m} / \mathrm{s}^{2}\right]$, respectively. Then the next condition is required to prevent the vehicle from going into a skid:

$$
\sqrt{a^{2}+a_{n}^{2}} \leq \mu g
$$

Here, for human-acceptable lateral acceleration limit, the next experimental model is proposed as a good approximation for urban driving (Bosetti et al., 2014).

$$
a_{n}\left\langle a_{\text {lim }}(v):=\frac{a_{0}}{\sqrt{\left(1-\left(v / v_{0}\right)^{2}\right)^{2}+2\left(v / v_{0}\right)^{2}}},\right.
$$

where $a_{0}$ represents the lateral acceleration limit when $v=0$, and $v_{0}$ represents the speed at the inflection point. The preceding study (Bosetti et al., 2014) employs $a_{0}=5.22 \mathrm{~m} / \mathrm{s}^{2}$ and $v_{0}=14.84 \mathrm{~m} / \mathrm{s}$ as suitable values for operations on the dry road, it is evident from Eq. (20) that the condition $a_{n}<\mu \mathrm{g}$ is required to enable longitudinal acceleration or deceleration. Thus, in this paper, $a_{0}=0.9 \mu \mathrm{g}$ is adopted while $v_{0}$ is set as $14.84 \mathrm{~m} / \mathrm{s}$, that is taken from the literature. The speed range is supposed as $0.0 \mathrm{~m} / \mathrm{s} \sim 16.7 \mathrm{~m} / \mathrm{s}(60 \mathrm{~km} / \mathrm{h}$ ). For $\mu=0.3$ (snow), 0.5 (wet) and 0.8 (dry), the relationship between $v$ and $a_{\text {lim }}(v)$ is shown in Fig. 6. It is observed that, as $v$ becomes higher, $a_{\text {lim }}(v)$ becomes stringent.

Substituting Eq. (21) into Eq. (20), the next inequality for (longitudinal) acceleration, which comes from the road friction, is obtained.

$$
-a_{\mu}(\mu, v) \leq a \leq+a_{\mu}(\mu, v), \quad a_{\mu}(\mu, v):=\sqrt{(\mu g)^{2}-a_{l i m}(v)^{2}}
$$

where the bound $a_{\mu}$ can be modeled by $\mu$ and $v$. For the same value of $\mu$ and range of $v$, the relationship between $\mu, v$ and $a_{\mu}(\mu, v)$ is shown in Fig. 7. As $v$ becomes larger, $a_{\mu}(\mu, v)$ is increased, thus the constraint is more relaxed. This result shows that, as the lateral acceleration limit becomes smaller, a larger longitudinal acceleration is allowed under Eq. (20).

This study intends to reshape the trajectory into a piecewise constant acceleration form depicted in Fig. 5; the soft constraint bounds for the acceleration can be chosen as rather conservative to a value at $v=0$ in Fig. 7. Configuring the constraint bounds as described, the proposed method becomes feasible by simultaneously considering the road friction.

\section{Experiment}

\subsection{Environment}

We conducted experiments on a straight road in a test course, with a packed snow surface. Hence we assumed the road friction coefficient as $\mu=0.3$. Figure 8 depicts the experimental vehicle which is equipped with additional computers and sensors to enable autonomous driving. GNSS receivers, whose positional resolution is a few centimeters in the open sky environment, are equipped to measure the vehicle's position and orientation. Several RADARs and LIDARs are also equipped to measure the distance between the ego vehicle and other vehicles. The RADARs are a mass production model 


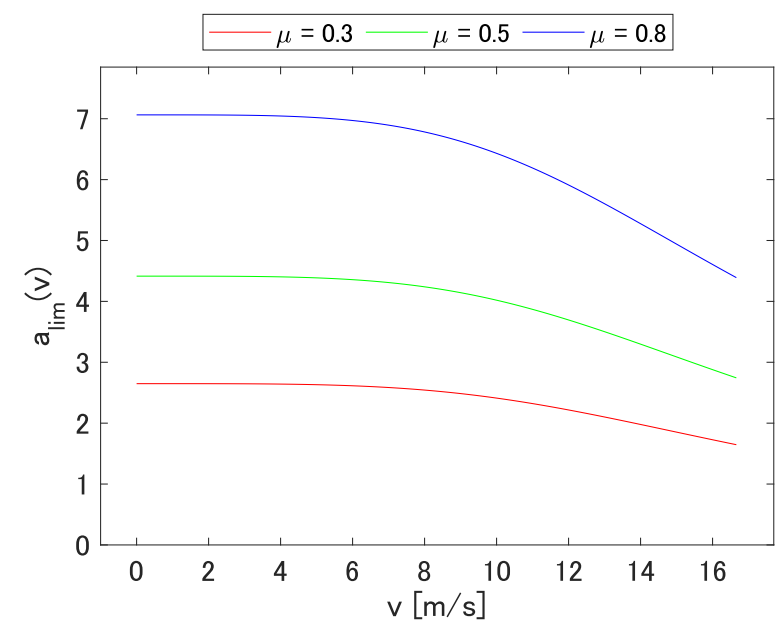

Fig. 6 Relationship between $v$ and $a_{\text {lim }}(v)$ (lateral).

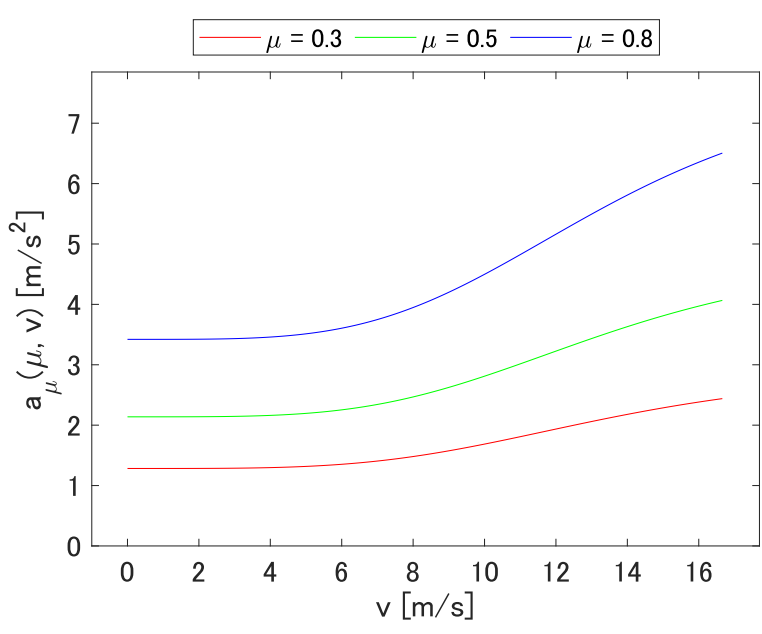

Fig. 7 Relationship between $v$ and $a_{\mu}(\mu, v)$ (longitudinal).

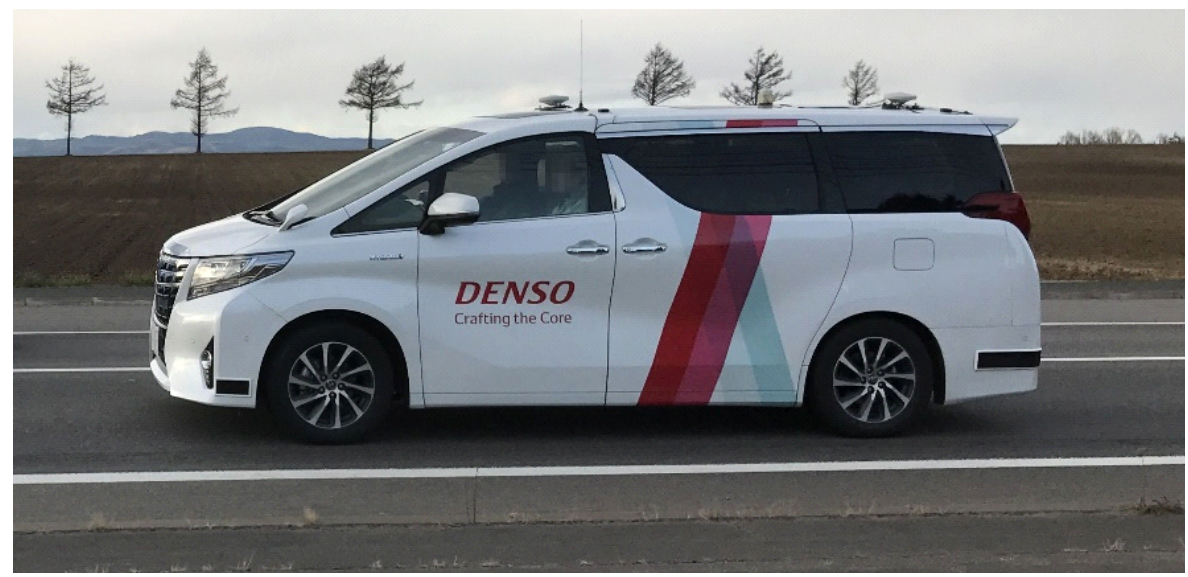

Fig. 8 Experimental vehicle.s Additional sensors and computers are equipped for autonomous driving.

and LIDARs are a commercial model whose range and resolution are $50 \mathrm{~m}$ and $0.04 \mathrm{~m}$, respectively. For the sensors to measure the vehicle's internal information, speed sensors and acceleration sensors are embedded. The proposed method is implemented as a real-time task with a $100 \mathrm{~ms}$ cycle in an industrial computer, which is placed in the trunk of the vehicle.

\subsection{Configuration}

Two experimental scenarios which require braking for collision avoidance are adopted to validate the effectiveness of the proposed method. The first scenario corresponds to sudden braking of the preceding vehicle, as depicted in Fig. 9 , while the second scenario corresponds to cutting-in by another vehicle, as depicted in Fig.10.

In the scenario corresponding to sudden braking, the vehicle initially tracks the preceding vehicle at $30 \mathrm{~km} / \mathrm{h}$, then the preceding vehicle suddenly decelerates by about $-0.15 \mathrm{gm} / \mathrm{s}^{2}$ until it stops, where $g$ represents gravity acceleration. In the scenario corresponding to cutting-in, the vehicle initially cruises at $40 \mathrm{~km} / \mathrm{h}$, then another vehicle travelling at $20 \mathrm{~km} / \mathrm{h}$ cuts-in. The distance between the vehicles at the cutting-in moment are set as two variants: about $35 \mathrm{~m}$ and $25 \mathrm{~m}$, and the reference acceleration trajectory for MPC is saturated by $-0.075 \mathrm{gm} / \mathrm{s}^{2}$ below to ensure the safety of the experiments. Through these experimental scenarios which require a large magnitude of braking, we validate whether the proposed method shapes the trajectory as well as avoiding collision.

In terms of parameters for the proposed method, the upper distance $d^{u}[k]$ was set as $5 \mathrm{~m}$ behind the preceding vehicle for collision avoidance. Then the maximum speed $v^{u}[k]$ was set as the speed limit of the test course. The lower distance and speed were set as a constant small negative value to avoid the vehicle receding. The other parameters were set as constant values, as listed in Table 1. Soft minimum acceleration $a_{s}^{l}$ is set to satisfy the requirement for road friction, described in Section 3.4. 


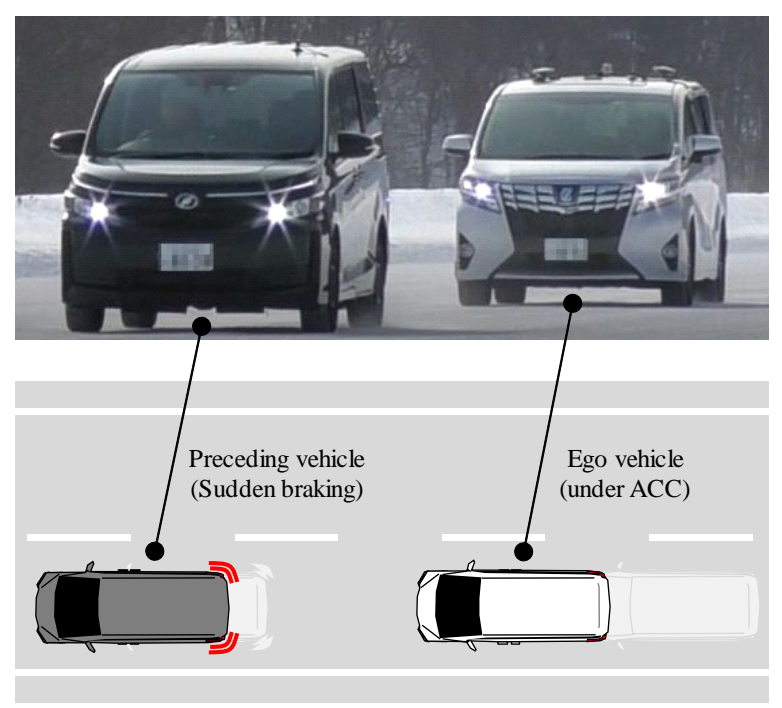

Fig. 9 Scenario 1: the preceding vehicle suddenly brakes and stops.

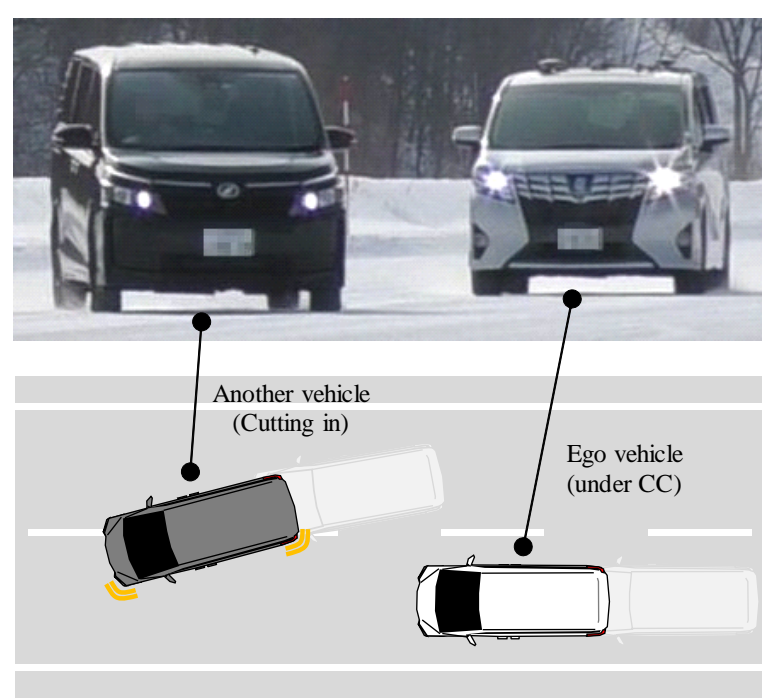

Fig. 10 Scenario 2: a slow-moving vehicle cuts in front.

Table 1 Parameter configuration for the experiments.

\begin{tabular}{lll}
\hline Parameter & Symbol & Value \\
\hline Hard maximum acceleration & $a^{u}$ & $1.471 \mathrm{~m} / \mathrm{s}^{2}$ \\
Hard minimum acceleration & $a^{l}$ & $-2.943 \mathrm{~m} / \mathrm{s}^{2}$ \\
Soft maximum acceleration & $a_{s}^{u}$ & $0.736 \mathrm{~m} / \mathrm{s}^{2}$ \\
Soft minimum acceleration & $a_{s}^{l}$ & $-0.736 \mathrm{~m} / \mathrm{s}^{2}$ \\
Soft maximum jerk & $j_{s}^{u}$ & $0.909 \mathrm{~m} / \mathrm{s}^{3}$ \\
Soft minimum jerk & $j_{s}^{l}$ & $-0.500 \mathrm{~m} / \mathrm{s}^{3}$ \\
Weight for the states & $\boldsymbol{Q}$ & $\operatorname{diag}(0.002,0.05,0.1)$ \\
Weight for the input & $R$ & 0.005 \\
Weight for acceleration slack variable & $Q_{a}$ & 60 \\
Weight for jerk slack variable & $Q_{j}$ & 90 \\
Number of the prediction points & $N$ & 30 \\
Prediction interval & $\Delta$ & $7 / 30 \mathrm{~s}$ \\
\hline
\end{tabular}

\subsection{Result and Discussion}

\subsubsection{Sudden braking}

Figure 11 shows the response of the ego-vehicle when the preceding vehicle braked suddenly. Figure 11(a) is the travel distance, (b) is the velocity, (c) is the acceleration, and (d) is the jerk with respect to $t$ [s], in which the target trajectory profile, the hard and / or soft constraints, the optimal state or the input of MPC, and the actual measurements are drawn. For brevity, constraints which were not activated are omitted. The travel distance was obtained by numerical integration of velocity, while the jerk was obtained by numerical differentiation of velocity. The acceleration and jerk are smoothed by taking a moving average of 15 points.

The vehicle successfully stopped, maintaining an appropriate distance greater than the prescribed minimum distance, $5 \mathrm{~m}$. The computation time required to generate the optimal trajectory was at most $34.5 \mathrm{~ms}$, which was sufficiently smaller than the control cycle.

Figure 11(a) denotes that the vehicle stopped without collision, and its velocity decreased smoothly. The actual vehicle tracked the optimal trajectory with time delay less than $1 \mathrm{~s}$ and the response of the actual vehicle did not result in any unacceptable overshoot or oscillation. Figure 11(c) indicates that the reference acceleration dropped and reached $-1.2 \mathrm{~m} / \mathrm{s}^{2}$ to generate more substantial deceleration which was below the level of the soft constraint, $-0.736 \mathrm{~m} / \mathrm{s}^{2}$, but the optimal acceleration obtained by the proposed MPC was saturated at the soft constraint in the same shape depicted in Fig. 5. Figure 11(d) also indicates that the optimal jerk is restricted inside the soft constraints between -0.500 and $+0.909 \mathrm{~m} / \mathrm{s}^{3}$.

Thus, the acceleration trajectory had an appropriate shape, and the jerk was confined in the constraints while the hard constraint on travel distance is satisfied.

\subsubsection{Cutting-in}

Figure 12 presents results pertaining to the case where another vehicle cuts-in front of the ego-vehicle at a distance 


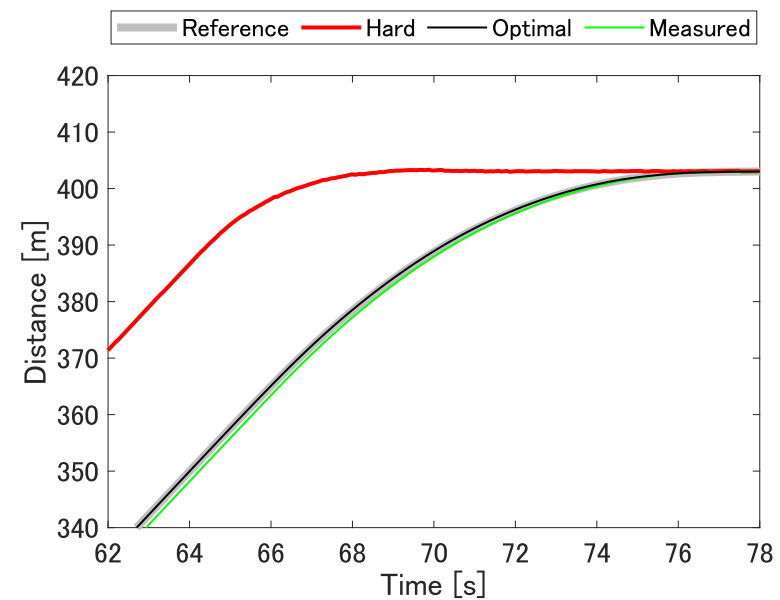

(a) Distance

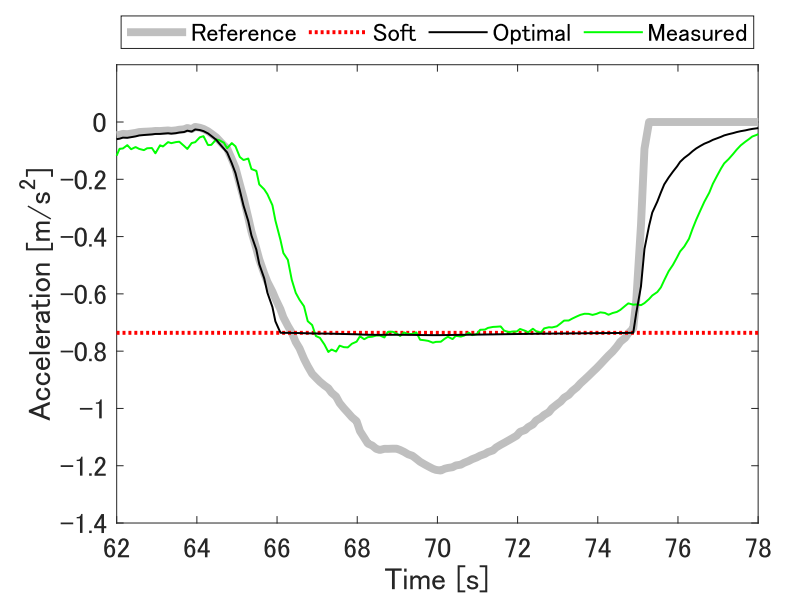

(c) Acceleration

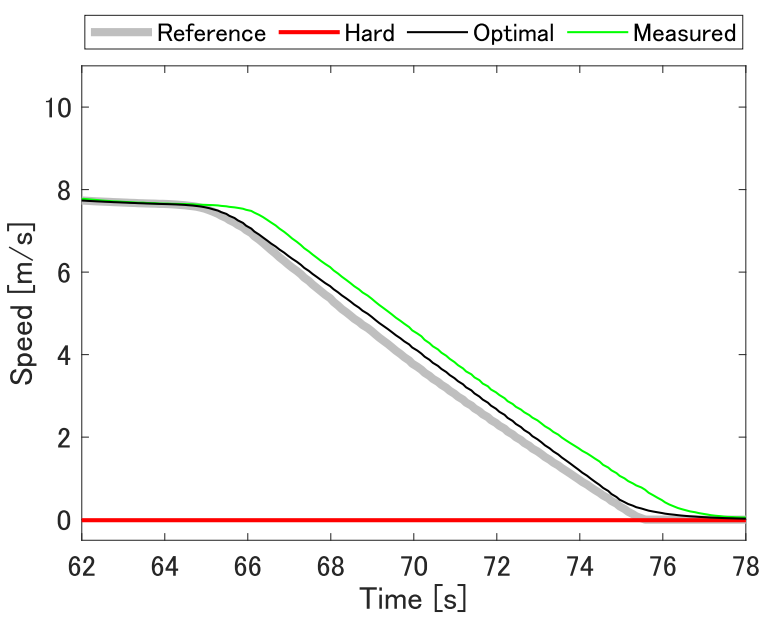

(b) Speed

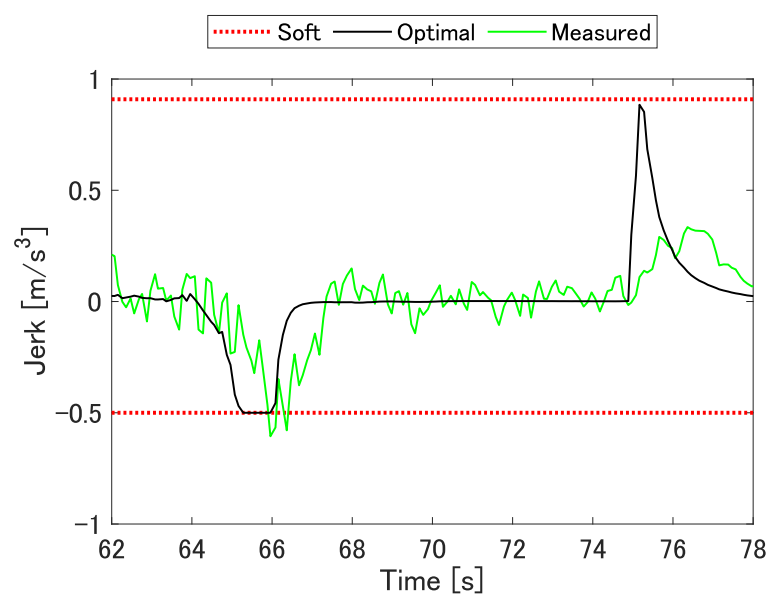

(d) Jerk

Fig. 11 Experimental results for scenario 1: the preceding vehicle for ACC suddenly brakes and stops. The reference acceleration exhibits substantial deceleration which has an apparent trough. The proposed method shapes the acceleration and jerk trajectories to what is desirable, while collision avoidance is achieved.

of about $35 \mathrm{~m}$. As depicted in Fig. 12(a), the ego vehicle approached the preceding vehicle, but it maintained sufficient distance, greater than $5 \mathrm{~m}$, by decreasing speed as depicted in Fig. 12(b). The maximum computational time required for both the integration of CC / ACC and MPC was $24.5 \mathrm{~ms}$, which was less than the control cycle. In this experiment, the reference acceleration was intentionally saturated as depicted in Fig. 12(c) to ensure the safety of the experiment.

Figure 12(a) indicates that the reference acceleration discontinuously changed when the preceding vehicle entered the same lane. So the reference acceleration trajectory also showed a discontinuous drop as depicted in Fig. 12(c). For the reference trajectory, Fig. 12(d) indicates that the jerk was shaped to satisfy the jerk constraint to be larger than $-0.50 \mathrm{~m} / \mathrm{s}^{3}$, which induced the acceleration slope in Fig. 12(c).

To ensure that the soft acceleration constraint can be relaxed if necessary, we conducted the same cutting-in experiment at a smaller inter-vehicular distance. Figure 13 depicts the results of this experiment with an inter-vehicular distance of about $25 \mathrm{~m}$. In Fig. 13(a), the hard constraint on travel distance dropped instantaneously at $t=7 \mathrm{~s}$. This was caused by the breeze of snow in front of the vehicle, but it did not affect the subsequent experimental results. Since the cutting-in distance was smaller than in the previous case, greater deceleration should be required. Figures 13(c) and 13(d) indicate that acceleration and jerk became smaller than the boundary specified by the soft constraints to obtain immediate and greater deceleration. This response was caused by the prediction to maintain an appropriate distance, because the relaxation appeared before the inter-vehicular distance became minimum at the duration $15 \sim 18 \mathrm{~s}$. These results confirm that the proposed controller can relax the soft constraints to maintain the distance margin required for safe operations. 


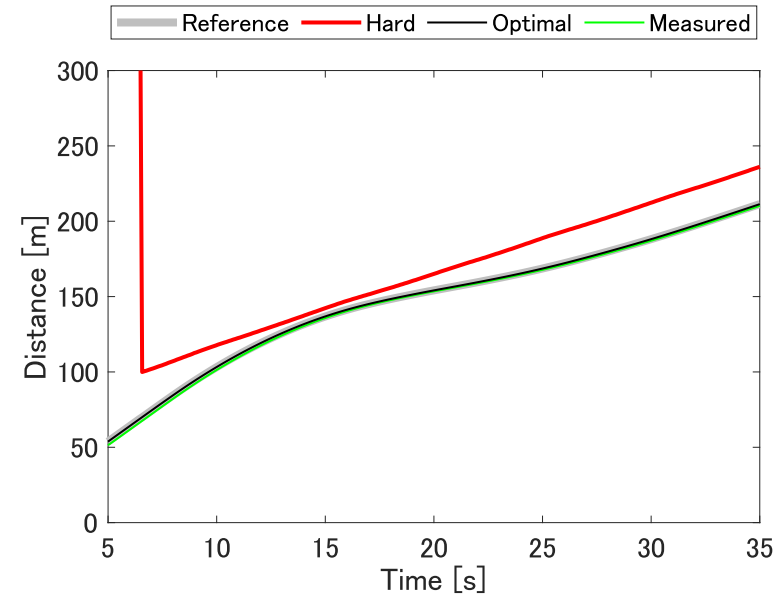

(a) Distance

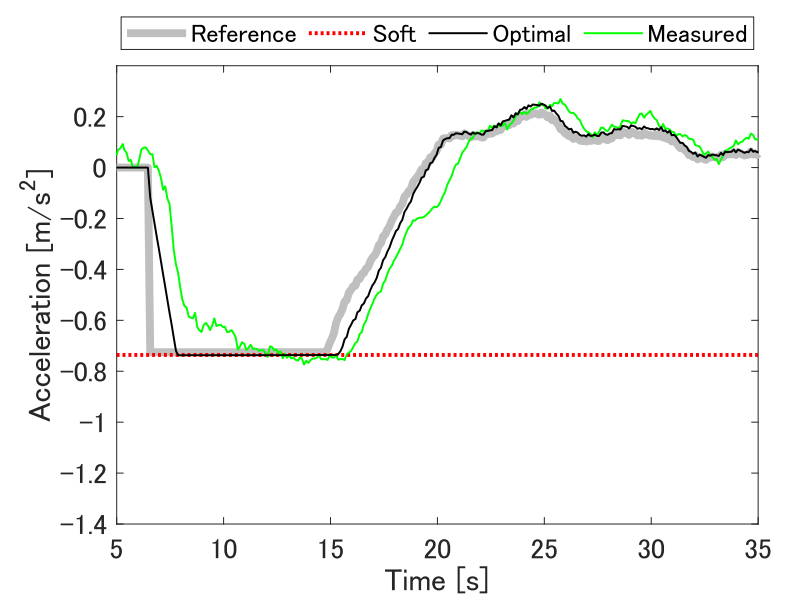

(c) Acceleration

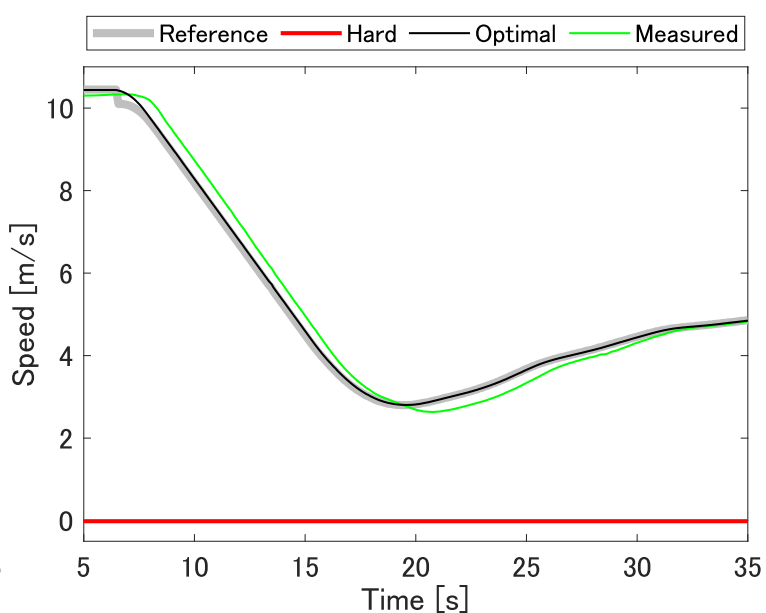

(b) Speed

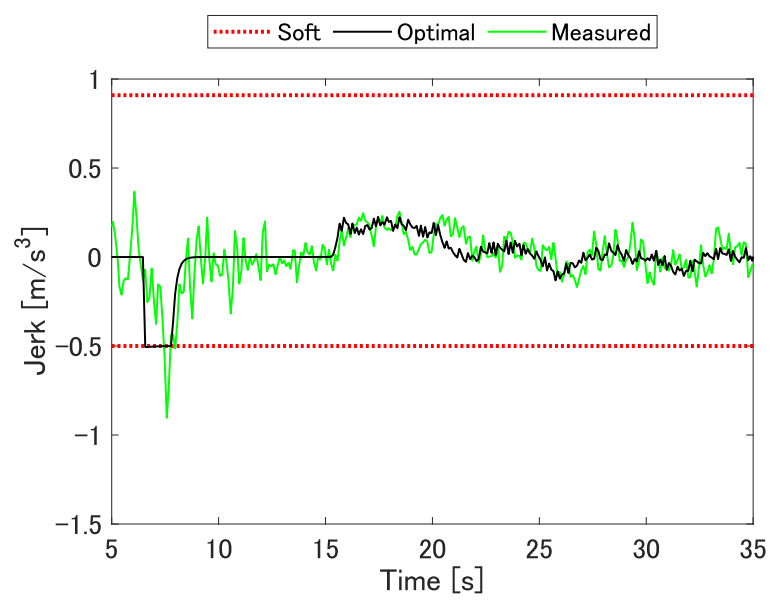

(d) Jerk

Fig. 12 Experimental results for scenario 2 (1): a slow-moving vehicle cuts-in on the front of the ego vehicle, with a relatively long margin. The reference acceleration suddenly and discontinuously changes when the slow-moving vehicle cuts-in. The proposed method shapes the jerk trajectory to the desired trajectory, while avoiding collision.

\section{Conclusions}

This paper focuses on acceleration trajectory reshaping using model predictive control for autonomous vehicles. The proposed method employs two types of constraints for this shaping: hard constraints which must be satisfied and soft constraints which can be relaxed. Utilizing the soft constraints, the acceleration trajectory is shaped into the desired piece-wise linear function of time, guaranteeing obstacle avoidance. We employ constant acceleration (deceleration) as a typical desired trajectory to verify the feasibility of the proposed method. The effectiveness of the method is validated through experiments with real vehicles in two scenarios in which braking for collision avoidance is required. The results suggest that the proposed method is effective.

Though the system delay shown in the experimental results did not cause practical problems, there is a possibility that proactive treatment is required. In this case, the proposed method can accommodate system delay in some manner if necessary. For example, actuator response could be considered by introducing additional jerk constraints. Further, communication latency can be compensated, utilizing the optimal predictive value for the target of the tracking controller. This feature is another merit of the proposed method.

Future tasks are as follows. Organoleptic evaluations to determine a preferable trajectory for passengers is required. Utilizing this result with the proposed trajectory reshaping method, the feeling quality and user's acceptability of the system might be improved. Since measured data are always accompanied by uncertainty, it is essential to explicitly consider the measurement uncertainty associated with obstacles. Enhancements in this respect offer great potential for 


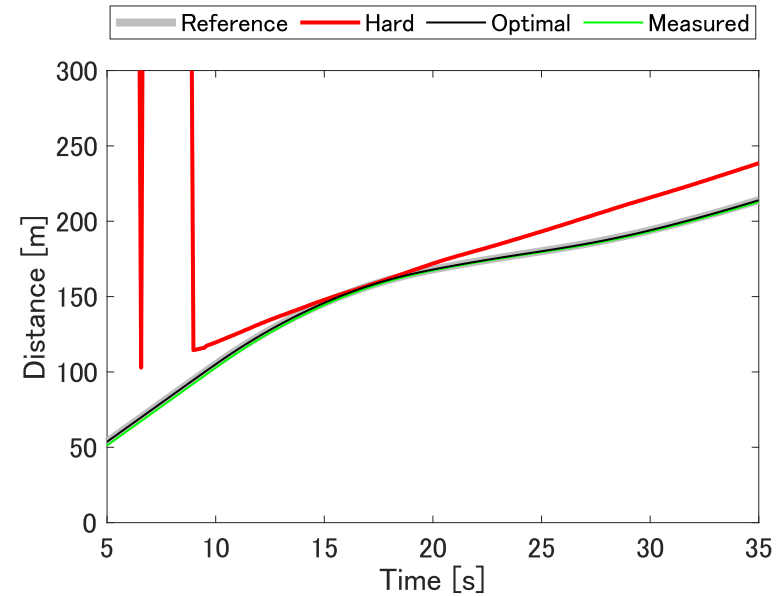

(a) Distance

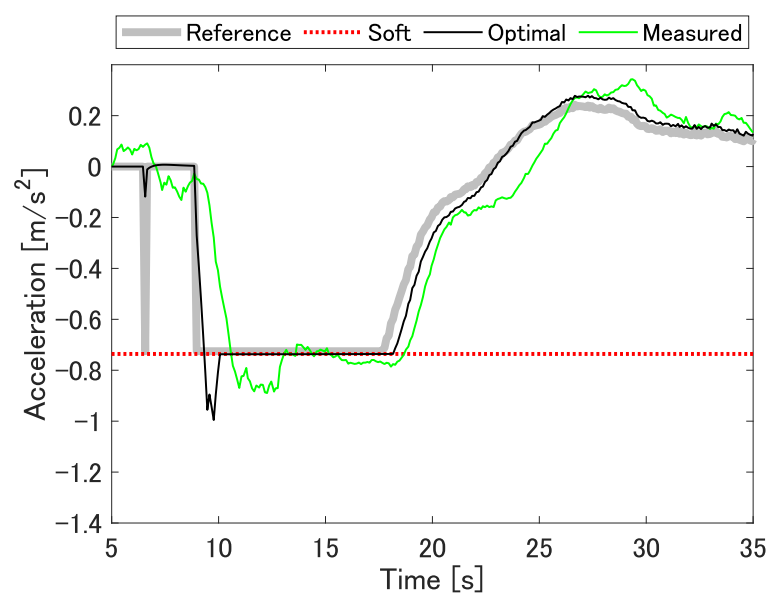

(c) Acceleration

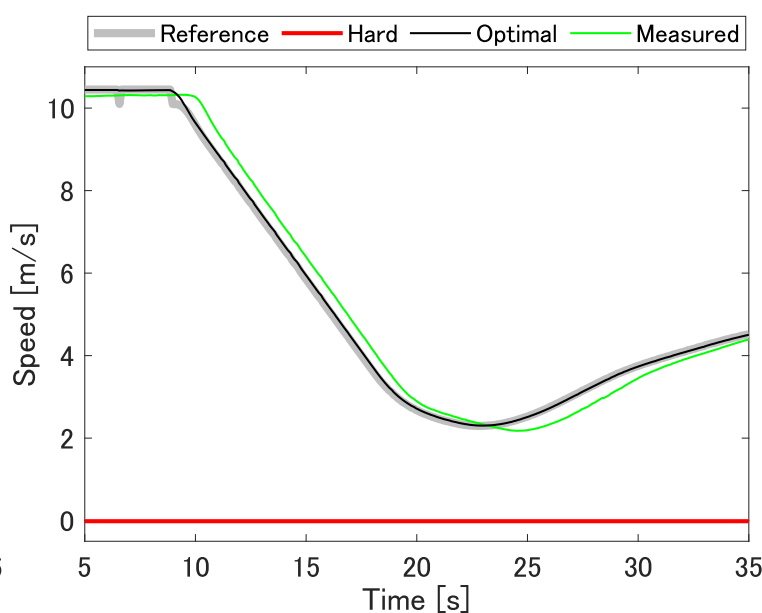

(b) Speed

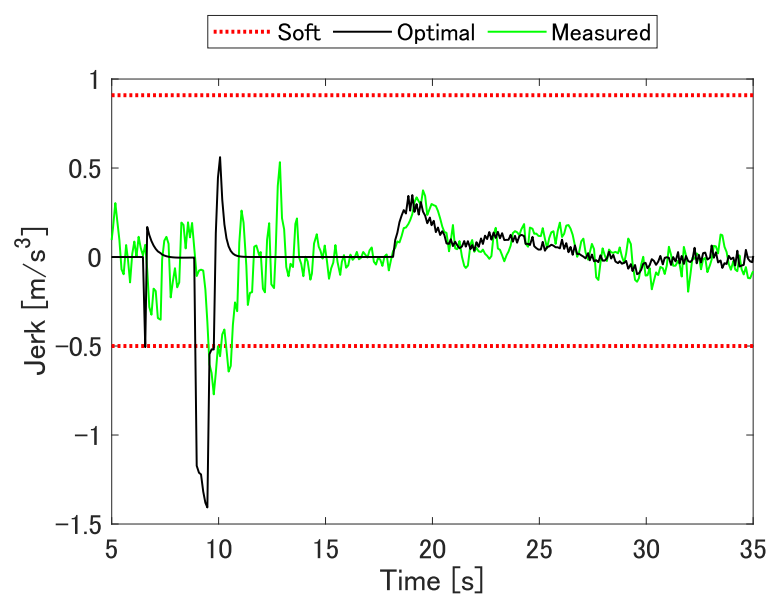

(d) Jerk

Fig. 13 Experimental results for scenario 2 (2): a slow-moving vehicle cuts-in front of the ego vehicle, with a relatively short margin. The generated trajectory is infeasible as long as the soft constraints for acceleration and jerk are satisfied. The proposed method relaxes the soft constraints to avoid collision, seeking to shape the trajectory whenever possible.

maximizing the safety of autonomous driving. It is also important to consider situations where there is no feasible trajectory. It is practically significant to generate a best effort trajectory, which provides maximal effort to avoid collision.

\section{References}

Aeberhard, M., Rauch, S., Bahram, M., Tanzmeister, G., Thomas, J., Pilat, Y., ... Kaempchen, N., Experience, Results and Lessons Learned from Automated Driving on Germany's Highways, IEEE Intelligent Transportation Systems Magazine, Vol.7, No.1 (2015), pp.42-57.

Bageshwar, V. L., Garrard, W. L. and Rajamani, R., Model predictive control of transitional maneuvers for adaptive cruise control vehicles, IEEE Transactions on Vehicular Technology, Vol.53, No.5 (2004), pp.1573-1585.

Bosetti, P., Da Lio, M. and Saroldi, A., On the human control of vehicles: an experimental study of acceleration, European Transport Research Review, Vol.6 (2014), pp.157-170.

Ioannou, P. and Xu, Z., Throttle and Brake Control System for Automatic Vehicle Following, IVHS Journal, Vol.1, No.4 (1994), pp.345-377.

Junhui, Z., Li, Q. and Chen, E., Integrated Adaptive Cruise Control with Weight Coefficient Self-Tuning Strategy, Applied Sciences, Vol.8, No.6 (2018).

Kianfar, R., Falcone, P. and Fredriksson, J., A control matching model predictive control approach to string stable vehicle platooning, Control Engineering Practice, Vol.45 (2015), pp.163-173.

Li, S., Wang, J., Li, K. and Zhang, D., Study on robustness and feasibility of MPC based vehicular Adaptive Cruise 
Control system, Proceedings of the 2009 IEEE Intelligent Vehicles Symposium (2009), pp.1297-1301.

Liu, K., Gong, J., Kurt, A., Chen, H. and Ozguner, U., A model predictive-based approach for longitudinal control in autonomous driving with lateral interruptions, Proceedings of the 2017 IEEE Intelligent Vehicles Symposium (2017), pp.359-364.

Maciejowski, J. M., Predictive Control with Constraints, Tokyo Denki University Press (2008), pp.99-101. (in Japanese)

Martinez, J. and Wit, C., A Safe Longitudinal Control for Adaptive Cruise Control and Stop-and-Go Scenarios, IEEE Transactions on Control Systems Technology, Vol.15, No.2 (2007), pp.246-258.

Mizushima, Y., Okawa, I. and Nonaka, K., Model Predictive Control for Autonomous Vehicles with Speed Profile Shaping, Proceedings of the 10th IFAC Symposium on Intelligent Autonomous Vehicles (2019), pp.31-36.

Naus, G., Ploeg, J., Molengraft, R. and Steinbuch, M., Explicit MPC design and performance-based tuning of an Adaptive Cruise Control Stop-\&-Go, Proceedings of the 2008 IEEE Intelligent Vehicles Symposium (2008), pp.434-439.

Okawa, I. and Nonaka, K., Comparative evaluation of a trajectory generator for obstacle avoidance guaranteeing computational upper cost, International Journal of Automotive Engineering, Vol.9, No.2 (2018), pp.39-47.

Persson, M., Botling, F., Hesslow, E. and Johansson, R., Stop \& Go Controller for Adaptive Cruise Control, Proceedings of the 1999 IEEE International Conference on Control Applications (1999), pp.1692-1697.

Saitou, Y. and Imura, J., An M-matrix based Efficient Algorithm for Model Predictive Control of Input Constrained Linear Systems, Transactions of the Society of Instrument and Control Engineers, Vol.40, No.9 (2004), pp.906-914. (in Japanese)

Stanger, T. and Del Re, L., A model predictive Cooperative Adaptive Cruise Control approach, Proceedings of the American Control Conference (2013), pp.1374-1379.

Vahidi, A. and Eskandarian, A., Research Advances in Intelligent Collision Avoidance and Adaptive Cruise Control, IEEE Transactions on Intelligent Transportation Systems, Vol.4, No.3 (2003), pp.143-153.

Wang, F., Sagawa, K. and Inooka, H., A study of the relationship between the longitudinal acceleration / deceleration of automobiles and ride comfort, Human Factors, Vol.36, No.4 (2000), pp.191-200. (in Japanese)

Yamamura, Y., Seto, Y., Nishira, H. and Kawabe, T., An ACC Design Method for Achieving Both String Stability and Ride Comfort, Journal of System Design and Dynamics, Vol.2, No.4 (2008), pp.979-990.

Zhao, R., C., Wong, P., K., Xie, Z., C. and Zhao, J., Real-Time Weighted Multi-Objective Model Predictive Controller for Adaptive Cruise Control Systems, International Journal of Automotive Technology, Vol.18, No.2 (2017), pp.279-292.

Ziegler, J., Bender, P., Schreiber, M., Lategahn, H., Strauss, T., Stiller, C., ... Zeeb, E., Making Bertha Drive - An Autonomous Journey on a Historic Route, IEEE Intelligent Transportation Systems Magazine, Vol.6, No.2 (2014), pp.8-20. 